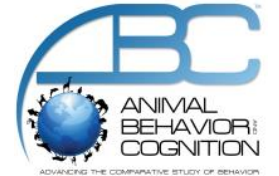

\title{
Doggy Vision: Examining how Dogs (Canis familiaris) Interact with Media using a Dog-Driven Proximity Tracker Device
}

\author{
Ilyena Hirskyj-Douglas ${ }^{1 *}$ and Janet C. $\operatorname{Read}^{2}$
}

\author{
${ }^{1}$ Department of Computer Science, Aalto University, Espoo, Finland \\ ${ }^{2}$ School of Physical Sciences and Computing, University of Central Lancashire, Lancashire, England \\ *Corresponding author (Email: ilyena.hirskyj-douglas@aalto.fi)
}

Citation - Hirskyj-Douglas, I., \& Read, J. C. (2018). DoggyVision: Examining how dogs (Canis familiaris) interact with media using a dog-driven proximity tracker device. Animal Behavior and Cognition, 5(4), 388-405.

https://doi.org/10.26451/abc.05.04.06.2018

\begin{abstract}
With screen technology becoming ubiquitously embedded into our homes, these screens are often in places where they can be viewed by domestic dogs (Canis familiaris); however, there is a lack of research showing to what extent, and for how long, dogs attend to media on screens. One pressing question is to understand if a dog, given the opportunity, would or could control its own viewing. This paper describes a prototype system (DoggyVision) that gives control to a dog in regard to the turning on and off of a TV screen in order to study activation with screen media in home settings. The system is used with two dogs to explore the interaction modalities between machine and dog. DoggyVision is shown to be non-invasive for the dog and easy to use in the home. Recordings show that dogs did attend to the screen but did not appear, in this study, to change their activation behaviors around the TV screen between being in no control (week 1), and in some control (week 2), of the TV media presentation. The study builds on 'dog-centered' methods to examine a dog's behavior non-invasively demonstrating that useful data can be yielded from dog-driven devices within the home. For the Animal Computer Interaction community, this is the first system that allows the dog to trigger the activation of the device as the system records the activation automatically.
\end{abstract}

Keywords - Animal Computer Interaction, Domestic Dog, Dog Computer Systems, Media and Dogs

Nonhuman animals (hereafter animals) have used technology for some time. The number of studies of how animals interact with technology, and of the impact of such interactions, has grown as computer scientists merge their interests with themes from ethology to form the new animal computing field coined Animal Computer Interaction (ACI, Mancini, 2013). This growth in research runs alongside an increase in consumer products for animals that include apps and media. ACI research has seen screen technology systems designed for animals for assistance technology (Zeagler et al., 2016) and entertainment (Smith, 2011; Westerlaken \& Gualeni, 2014). Advancements in technology have consequently allowed ethologists to further explore the human-animal relationship communication dialogue through directional cues such as gaze and gesture tracking (Lakatos, Gácsi, Topál, \& Miklósi, 2012; Soproni, Miklósi, Topál, \& Csányi, 2001).

Dog Computer Interaction (DCI) is the sub-field of ACI where the animals of interest are dogs (Canis familiaris). Given the large number of dogs living in domestic situations, understanding how they interact with technology, and with media, is especially interesting. Since 2009, the Californian TV 
channel, DogTV (2016), has claimed a growing audience (30 million) worldwide and this has prompted the desire to better understand what dogs watch and to what extent dogs might be able to choose content. Others interested in dog media behavior include those concerned with the welfare of dogs in homes. The 2015 People's Dispensary for Sick Animals (PDSA) animal wellbeing report (a study of animals in the UK) suggested that, out of a UK population of 9.3 million dogs, on working days, over 3 million were left alone in their owners' homes for quite a considerable length of time (PDSA PAW Report, 2015). This suggests that appropriately designed technologies for dogs in the home could be societally beneficial. For the research community, the study of such technologies can provide important insights into the design of user interfaces for dogs and can contribute insights towards the dogs' experiences.

Humans' behaviors and viewing habits with screens and media devices are typically investigated by using self-report methods (surveys), using logged data of channel and content selection activities, or by the use of tracking systems that capture what is being watched (Abreu, Almeida, \& Teles, 2014; Raynor et al., 2013). In ACI, self-reporting can be done only where there is a proxy for the dog (typically an owner or animal behaviorist) and this is not ideal to study dogs interacting with screens when not with owners. The use of selection of channels as a method for study is currently not possible as the dog cannot (to date) select a channel. This leaves the use of tracking of vision and attention as a candidate method. Tracking studies are used in animal science and, as they use measurements directly from the dog, with careful design can be very dog centric. Animal centric (and by association, dog centric) research is a main theme within ACI as the community tries to focus on research and design that does not require the animal to change its natural behavior (Hirskyj-Douglas \& Read, 2014; Mancini, Lawson, \& Juhlin, 2016). Animal centric design, similar to HCI's user centered design, therefore aims to design technology that fits the animal's context and minimizes any need for the animal to be trained.

In this paper, we present a study describing and evaluating an automated screen device for dogs, DoggyVision, that uses body tracking to allow researchers to investigate a dog's behavior with a media screen in a home setting over a period of time. The DoggyVision system is shown to be useful to track system activation behavior and has potential to also be useful to explore what might happen if it were possible to give control of a device, to a dog.

\section{Motivation for Dog-Driven Systems}

The impetus for a dog-driven media system is to explore how dogs might use future interactive screen-based devices, especially in unsupervised ways. Systems, like the one presented here, could provide entertainment for dogs left alone or could provide a method of communication between the dog and screen. Such a system would represent a move away from the traditional paradigm of using technology with animals with the animal being an unconscious or coerced user, towards positioning the animal as an active 'consenting' user within the animal centric paradigm. We take a known HCI method of a proximity detected activation area and implement this concept with dogs ensuring a good fit to the end-user's (the dog's) requirements. In transferring methods from HCI to ACI we are able to contribute methodological knowledge to both fields. Additionally, this paper brings together animal behavior and computer science research assisting in developing a more conjoined body of knowledge as laid out within the ACI Manifesto (Mancini, 2013).

\section{Aims and Predictions}

Dogs have long been assumed to attend to visual media and recent studies using eye-gazetracking have shown this to be the case (Somppi, Törnqvist, Hänninen, Krause, \& Vainio, 2012; Williams, Mills, \& Guo, 2011). Such technology has demonstrated that dogs have similar gazing behaviors as humans (Törnqvist et al., 2015). Given this background, this paper seeks to discover, as its first aim, whether there is a technological solution that could give a dog some control over played media. 
Secondly, the aim is to examine whether, given such a system, a dog would show evidence of learning how to use such a system without human training.

We predict that a technological system can be designed and developed but also that it will have some limitations, especially given the huge variety in the size/weight/behavior of dogs and the variety of home settings that exist in the context of home screen viewing. The second prediction is that, if a dog could control the turning on and off of media, then over time, the dog would learn to actively control content. This would be demonstrated in the system described here as an increased tendency to activate or avoid the system over time.

\section{Method}

The study is presented here in two parts - firstly we describe the DoggyVision system build then we present the field research section, where the DoggyVision system was placed in homes for two weeks and data was gathered in use.

\section{System: DoggyVision}

Design and ethical decisions. In designing this system, it was important to consider how DoggyVision would be used, both as a research tool but also as a system prototype. In Issa and Isaias's model (Issa \& Isaias, 2015), HCI is modeled to (1) Interact with and through technology (2) Seek to understand and support human beings. These two represent (1) the functionalities of a system and (2) the emotional needs / experience needs of a user. HCI therefore aims to look at the function of systems (i.e., in the case of DoggyVision, the positioning of the sensors, how the system would work, how the system data could be analyzed) and the deeper experiences of the user, including emotional needs (in the case of DoggyVision, this refers to the choices to be made in terms of the media content and dog enjoyment, Lim et al., 2008).

Functionally, the DoggyVision system was required to play pre-selected media when a tracked body (the dog in this case) was detected in front of the screen. On detecting a tracked body, the system was additionally required to record the activation of the user with the screen and package this in such a way that it could later be analyzed. The data was required to be saved in a log both locally and online to allow for remote inspection of the DoggyVision activation. The log needed to contain information on when the user was in the sensed area (time, date and device) and the length of activation.

In terms of meeting the dogs' emotional needs, certain additional design requirements were specified. The first of these was to build a system that did not need the dog to be trained. This approach was chosen to allow the dog to freely choose how to use the system from his or her own affordances, stepping away from the human influenced behaviors where dogs have been shown to learn behaviors (Pongrácz et al., 2001) or copy novel actions (Topál, Byrne, Miklósi, \& Csányi, 2006). Secondly, we decided that allowing a dog to choose its own way of "interacting" also allowed for the dog to be able to walk away and not use the system, allowing a form of "consent" within ACI (Hirskyj-Douglas \& Read, 2016b; Mancini, 2016).

Hardware and software. The DoggyVision system is comprised of the hardware, the software, and the installed media. In theory, the system can be used with any screen and computer, but, in this case, the hardware used was a single monitor (HP L9600) positioned at head height for the dog (HirskyjDouglas, Read, \& Cassidy, 2017), a webcam (Logitech HD720p) positioned above the screen in a central position to capture the activity in and around the activation area, two PC speakers (creative SBS 260) to output sound with the volume set to $25 \%$ (to be audible but not so loud as to frighten and cause distress to the dog), and an Arduino tracking/sensing device positioned at the dogs' body height (Figure 1).

The software created for the DoggyVision system was written in Java. This controlled the delivery of media clips (FLV files), the recording and processing of the Arduino input from the sensors, 
the local and online saving of status reports, and the recording of system activations through video recordings (Figure 2).

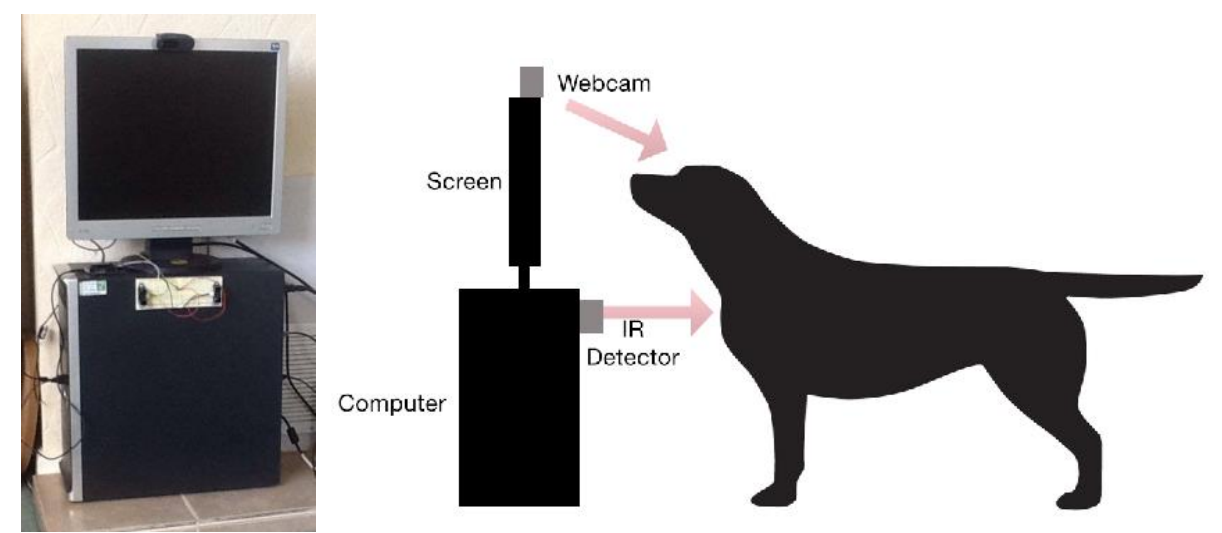

Figure 1. DoggyVision setup. Left image shows DoggyVision within the home and right image shows the setup through the detection mechanisms.

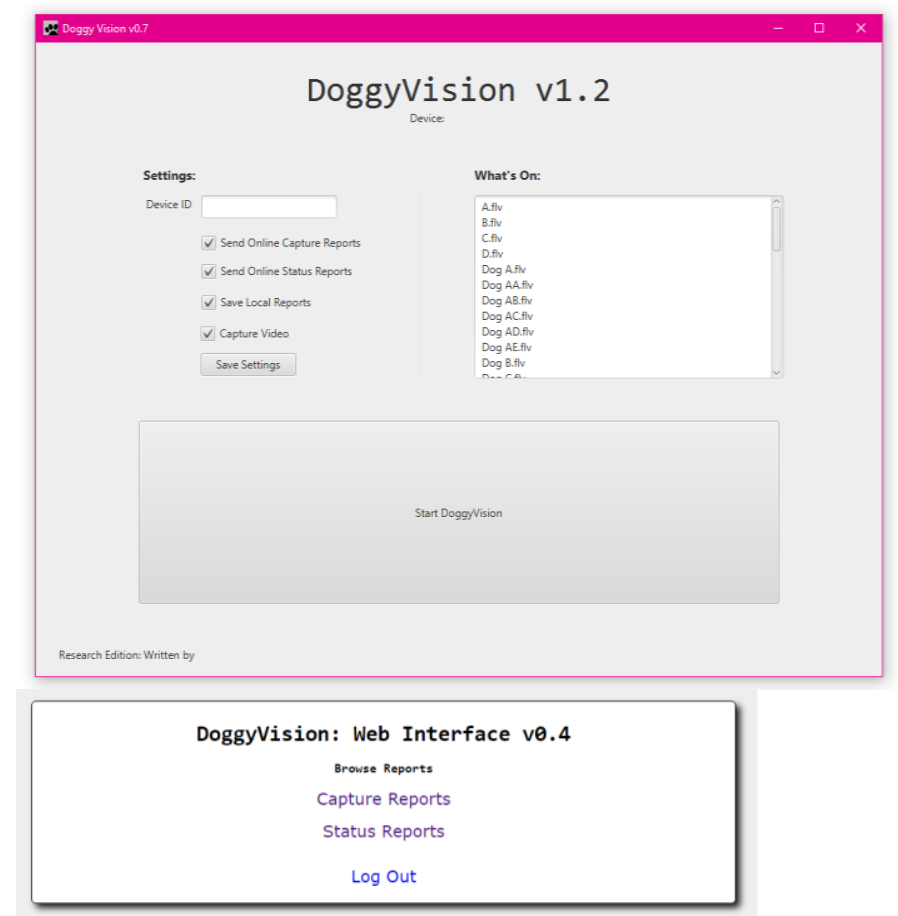

Figure 2. Main menu screen of DoggyVision v1.2 showing options and media clips with second picture displaying the web interface, which recorded capture report details and device status reports.

Media clips. Previous research has investigated dogs' preferences in regard to visual media (Somppi et al., 2012; Törnqvist et al., 2015; Williams et al., 2011) and has shown that dogs, given the choice, will pay greater attention to media that shows other dogs. For this reason, DoggyVision was installed with 39 media clips that each showed a range of different dogs in different situations. These media clips had previously been shown to an even-tempered dog that showed no distress or negative behaviors when viewing them. For the purposes of the research study, when activated, the DoggyVision 
system chose media clips from the 39 clips at random (using a software function) to minimize effects of the media content on the dogs' attention habits.

DoggyVision tracking. There were several possibilities for the design of the tracking system. Gaze tracking was certainly going to be unobtrusive and easy for the dog, but it was discounted due to the prohibitive cost of setting it up for multiple dogs and because of difficulties with accuracy. Posture tracking (using cameras to see how the dog was positioned) was also a possibility; some recent work has been undertaken on semi-automatic posture recognition (Mealin, Domínguez, \& Roberts, 2016) and head positioning (Hirskyj-Douglas, Huailan, \& Read, 2014) but these systems need many images to train the recognition process and so can be costly in time to set up and may cause discomfort for the dogs when building the training catalog. Infra-Red (IR) tracking, which relies on the heat given off by the dog, was an un-intrusive option. There were two choices for IR tracking, using motion sensors and using distance sensors. Although IR motion sensors work well in laboratory settings, we found during piloting that dogs would often pause to watch the screen, and this inertia caused the DoggyVision system to turn off, even though the dog was seen to be still attending to the screen. IR range detectors allowed for the dogs' natural behavior (of close proximity to the screen) and facilitated interactivity detection.

The DoggyVision tracking device therefore combined an Arduino microcontroller (Leonardo) with two IR distance sensors (Sharp GP2Y0A02YK) (Figure 3), to create a $100 \mathrm{~cm}$ by $20 \mathrm{~cm}$ (horizontal) activation area in front of the screen. The IR distance detectors were placed $15 \mathrm{~cm}$ apart to allow for the largest activation range, to try and ensure an experience for the dog that allowed the media to play without constantly turning on and off and also allow the dog to behave without constraint with the system. As DoggyVision used two readers, to improve proximity accuracy, the readings were averaged out over the last 25 readings to make sure the readings were within a $93 \mathrm{~cm}$ range of each other, following Doggen's (2014) Arduino Sharp IR guidelines mitigating against false readings.

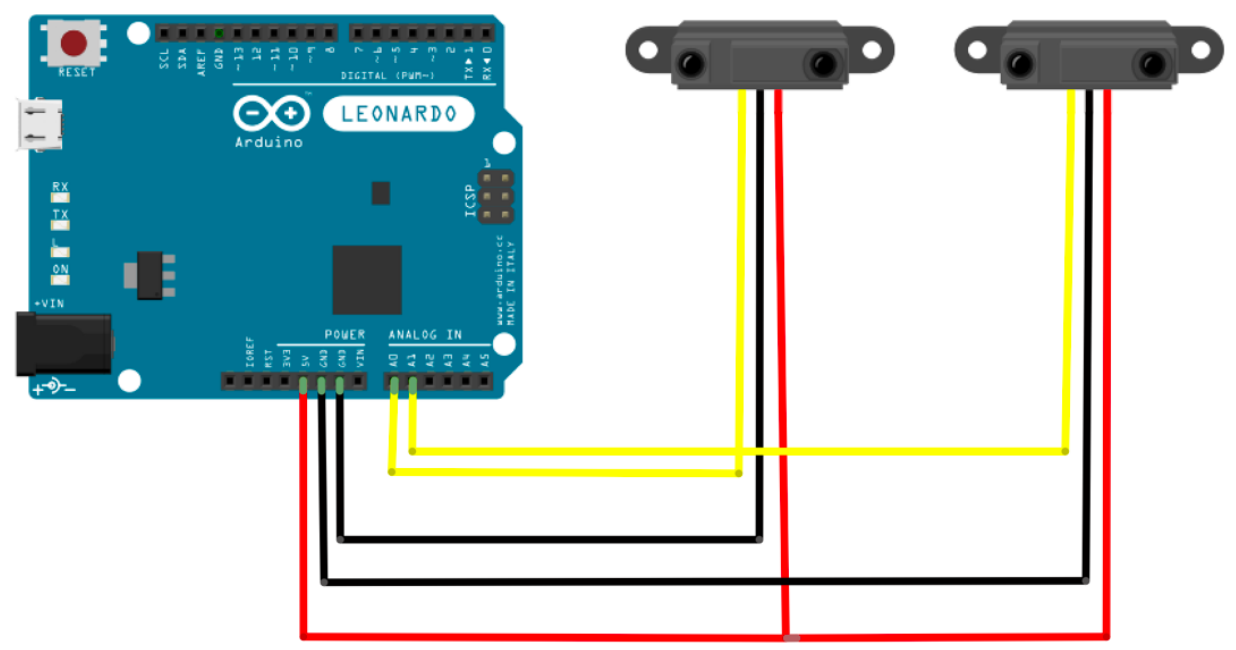

Figure 3. Setup used for DoggyVision Infrared (IR) range detecting with two Sharp 2 Y0A2 range finders. ${ }^{1}$

Research considerations. The dog moving into the tracked area was modeled in the software as a keypress event (this is how an input to a program is typically expressed). There was a very slight delay (and the delay also slightly varied according to the position of the media being played) between the keypress being detected by the DoggyVision tracking device and the respective media clip being selected, loaded into memory and played on the screen. For consistency in analysis, the media activations recorded

\footnotetext{
${ }^{1}$ The Arduino code used can be found at the author's website: https://ilyenahirskyjdouglas.wordpress.com/2017/09/01/doguino/
} 
by the DoggyVision software were for the duration that the media clip was being played on the screen rather than the timings of the keypress.

Fine-tuning the operation. In line with user-centered design principles, and to ensure that the system was both fit as a research tool but also as a design / system prototype - the built system was pilot tested with one $\operatorname{dog}(\operatorname{Dog} \mathrm{A})$. During the piloting, it became apparent that the way a dog moves in front of a proximity device was very different from human movement. For instance, a dog's tail wag movement occurring in the sensed area, could result in the dog being rapidly detected and undetected (i.e., tail vs. no tail). These on/off inputs were too quick for the software to process (it had to act, log and save each activation) and caused the system to crash. Taking from our previous research (Hirskyj-Douglas et al., 2017), that reported dogs' average screen interactions, the software was adjusted to work with three second intervals of activation. Once activated, the system stayed as was for three seconds- at the end of the three second period, the software checked for a user (dog) within the activation area and responded accordingly; thus, if a user was there, the media continued for another $3 \mathrm{~s}$. This resulted in activation lengths that were multiples of three seconds, viz. 3, 6, 9 etc. of media playtime hereon referred to as media iterations. A media activation is defined in this context as a single activation of the DoggyVision device by a dog, with each activation potentially being made up of at least one, but possibly many, media iterations.

The DoggyVision system was positioned to suit the individual dog by calibrating it to track at a height associated with the dog's biggest body mass, in this case, the dog's body (chest) height. Placing the tracking this high allowed the dog to sleep (lay down) within the space without continually activating the DoggyVision system. This was one design feature of the system that demonstrated that the DoggyVision system was designed to be dog friendly. One limitation of the implemented system was that it was possible for a dog to attend to the media and be outside the tracking field - in which case activation would end. However, when the system was in use, this situation was seldom seen. Another limitation was that the researcher was unable to fully know if the dog 'understood' the actions; i.e., whether it could deduce that staying in/entering into the tracked area caused the media to play as a similar study to this had not been conducted prior. However, as stated within our aims, this behavior was to be investigated within this study.

Classification of interaction and activation. Within this work, a media activation is an action that results from the dog being identified within the tracking space. It can be suggested that, at this point, the dog may be interacting with the media. The term "interaction" however, needs some explanation. It is acknowledged, in the sense of Buchanan's (2001) definition of interaction, that the degree to which a dog can meaningfully interact with a computer system is unknown as dogs' intentions, and what dogs perceive as possible to do within a computer system, are unidentified. This is not to imply that dogs cannot have implicit or unaware interactions that are meaningful, but that the scope behind terming what is meaningful to a dog within the interaction is unknown. In computer science, HCI refers to interaction through archetypical structural theoretic frameworks with the most used being from Norman (1998) who modeled interaction in terms of the user executing an interaction and then evaluating the result of that interaction. Core to Norman's model is the notion of intention; the user has a goal, interacts, sees the results of the interaction and perceives if the goal has been reached. Central to Norman's model is the idea that an interaction may have breakdowns that may or may not be discerned. For example, the user may interact, meet the goal but may not understand how the goal was reached and thus may not be able to replicate it. Errors in interaction are classified as mistakes (the user doing the wrong thing) or slips where the correct action was intended but a slip is made (accident). One main problem in applying this model to animals is that the animal's intention is hard to discover and so it is hard to discern if an interaction is deliberate or accidental. In ACI, researchers have sought to discover a dog's interactions by studying the dog but this is a very incomplete science (Geurtsen, Lamers, \& Schaff, 2015; Mancini, 2016; Zeagler et al., 2016). There may be possibilities to make a reasonable guess at a dog's intentions in systems in which the dog is trained, as suggested by Freil et al. (2017). 
Given the problems with applying human centered models to ACI, the term "interaction" is considered here in a broad sense, to include: the animal controlling a system (Britt, Miller, Waggoner, Bevly, \& Hamilton, 2011; Ritvo, \& Allison, 2014; Robinson, Mancini, Van Der Linden, Guest, \& Harris, 2014; Zeagler et al., 2016), systems that detect an animal's behavior (Majikes et al., 2017; Valentin, Alcaidinho, Howard, Jackson, \& Starner, 2015), systems that react to an animal's behavior (Geurtsen et al., 2015; Mancini, Harris, Aengenheister, \& Guest, 2015) and systems in which the animal interacts with a human (Lee et al., 2006; Lemasson, Duhaut, \& Pesty, 2015; Mealin et al., 2015; Morrison, Møller, Manresa-Yee, \& Eshraghi, 2016; Resner, 2001; Trindade et al., 2015). As noted within the ACI manifesto (Mancini, 2011), the interaction can also be explored by improving the usability of systems as well as by creating a meaningful experience for the animal. This is evidenced in work that has studied how animals can have an input into technology (Mancini et al., 2015; Perdue, Clay, Gaalema, Maple, \& Stoinski, 2012; Robinson et al., 2014; Zeagler et al., 2016), how they can be soothed or stimulated by technology (French, Mancini, \& Sharp, 2015; Geurtsen et al., 2015) and how animals and humans can be connected through technology (Carter, Webber, \& Sherwen, 2015; Lemasson et al., 2015; Mealin et al., 2015; Morrison et al., 2016; Resner, 2001).

Our aim was to create a system that reacted to a dog and allowed the possibility for the dog to control the system, using behavior as an activation instigator, with the goal to provide meaningful experiences for the dog.

\section{Field Study: DoggyVision in the Home}

The remainder of this paper describes the use of DoggyVision with two dogs. As this is the first study to enable dog directed untrained activations of a computer system within the home, contributions come from the data and the discussion around the approach in terms of its usability in dog computer systems.

As a research tool, the DoggyVision system was placed inside the dog participant's home for two weeks. It was adjusted to be operated in two modes; mode one, in which the system tracked the dog's behaviors but did not respond (i.e., no media was played) and mode two, in which media was additionally played as the dog entered the tracked area. In both cases, the system recorded video of the activity in front of the screen when a human or dog was within the activation space. For the first week of use, the system was in mode one and then in the second week, in mode two.

With technologies, such as home-based media devices, for instance TVs and computer screens, laboratory experiments are not appropriate as they do not take account of the way people, and animals, live (Olsen, Sellers, \& Boulter, 2014). Installing the device in an owner's home allowed for the dog to interact in a relaxed way and also allowed for a lengthier study than would have been possible in a laboratory. The first week of installation was used to create a baseline, which was then compared against the second week when the DoggyVision system played media clips as the dog approached. Baseline data has previously been shown to be useful in animal behavior studies as animals vary enormously in their activity levels (Piotti, Spooner, Jim, \& Kaminski, 2017). In this way, the study was dog friendly, required no training and did not gather trained responses; it also allowed the captured data to come directly from the dog end-user.

The DoggyVision system ran without interruption in the home for two weeks, in the two different modes, and then owner observations on the dog's use of the device were gathered to assist in the interpretation of the activations. The dog owners were asked open-ended questions to describe their dog's experience, but it should be noted that the primary method of data collection was from the dogs themselves. A survey could have been used with the owners but the main aim of DoggyVision was to establish what could be detected from the dogs themselves and especially from the 'all-day' unsupervised use of the DoggyVision system.

Subjects. Two dogs were involved in this study. As different breeds of dogs have varying physiological structures impacting upon their senses (vision and hearing) both dogs included in the study were from the same breed, the Labrador. Both dogs were Kennel Club (KC) registered. According to their 
owners, and according to a qualified veterinarian, the two dogs included had no behavioral or visual problems. Dog A was a neutered 61-month male Labrador and Dog B was a neutered 24-month old male Labrador. Dog A was $136 \mathrm{~cm}$ and Dog B $120 \mathrm{~cm}$ long from nose to tail with Dog A being $22 \mathrm{~cm}$ at its thickest point (activation point) and Dog B being $18 \mathrm{~cm}$. It should be noted that the DoggyVision system was 'set up' specifically for this size/type of dog. This was necessary and does speak to the limitations of any possible design as predicted at the outset of the study.

In line with the ethical principals in ACI (Hirskyj-Douglas \& Read, 2016b; Mancini, 2016), and to provide as ordinary setting as possible, the studies were conducted in the dogs' own home environments. Any locational variability of the study settings (between the two dogs) was mitigated by the study design, by which the dogs' behaviors with the track and play mode (mode 2) were compared with their activities in week one (mode 1), rather than them being compared across study locations.

The system was installed in the homeowner's lounge, which the dog had constant access to, even when the owner was out. For each dog, this room was a similar size (approx. $6 \mathrm{~m}^{2}$ ) and it included the TV and the dog's bed. The system was placed where the TV would normally be located. In this way the system was not in a place where the dog would accidentally activate it through its normal walking activity around the house (i.e., not in a corridor nor in front of a door). For each dog, the dog was the only dog in the house, and there were no children. Both owners, who lived alone with their dogs, worked for two days a week and actively left the home for $10 \mathrm{hr}$ during this period, otherwise they resided in the home with the dog. Each dog was left alone for around $30 \mathrm{hr}$ each week.

It is worth noting that, throughout the study, the dogs could walk away from the DoggyVision system whenever they wanted and could therefore effectively choose to not interact with the DoggyVision system. This enabled a form of consent for the dog in terms of his or her participation in the study. The daily activities of the dogs and their owners, such as walks, were kept similar during the two weeks to reduce the variabilities between the weeks. This daily activity included the amount of time the owner left the dog alone and the amount of exercise the dog had. Dog A and Dog B were left at home with the device approximately $90 \%$ of the time, with each getting an hour's walk daily.

Data analysis. When the system was activated, the data was stored locally and also sent to a server. In this way, any lags in storing the data and any system failures would have been noted as the researcher could remotely check the DoggyVision system. Over the study period there were no system failures.

The process of data analysis required three stages: (1) cleaning and verification, (2) coding, and (3) comparisons across modes. To begin with, the data was cleaned and verified by analyzing the recorded local and online time/interactivity data against the captured video recordings associated with each activation timing. This bringing together of the two sets of data was necessary to ensure that the activation gathered was initiated by the dog, and not by the owner. Activations caused by non-dog triggers and their associated recorded videos were removed. Following this stage, the data that remained was considered to be made up of a series of 'dog-activations', where an activation represented the triggering of the DoggyVision system by a dog who was within the detection range.

In the second phase, coding, the recorded video associated with each dog-activation was analyzed to determine whether the dog was looking at the screen. 'Not looking' viewing was defined as when the device detected a dog within the activation space, but the dog was not actively seen to attend to the screen. 'Looking' was defined as a dog being seen to be oriented towards the screen at any time during the total activation period. This coding scheme was verified by having three researchers analyze a small sample of recorded videos (5 from Dog A; 15 from Dog B) from week 2. These sampled recorded videos were chosen at random using the Haahr (2016) random number generator and the inter-rater reliability was analyzed using joint probability of agreement.

Having coded the data, comparisons were made within dogs (week 1 vs. week 2), and between $\operatorname{dogs}$ (Dog A vs. Dog B). Counts were then made of the number of activations (media iterations), the frequencies of the looking and not looking coded activations, the activation lengths (in terms of the number of continuous media iterations played by the DoggyVision system), the range of activation 
durations and the total activation time (over week 1 and week 2). When interpreting the data, it is important to understand what could be happening. For example, when there is a three second logged dogactivation (one media iteration play), this could either represent a single activation, where the dog enters and then leaves the tracked area before the end of the media clip, or a multiple activation, where the dog might enter, leave and then re-enter and leave (although this would be quite hard for a dog to achieve within a three second period and it was not seen when the videos were examined). In a longer dogactivation (e.g., 6 or $9 \mathrm{~s}$ ), it could be that the dog entered and remained (leaving at some point in the last 3 $\mathrm{s}$ thus initiating the end of the media stream), or that the dog entered and left and re-entered in such a way that the leaving and re-entering was within a three second media clip and thus, it would appear that the dog was always there. Again, rapid entering and leaving was unlikely given that the timings were so short - and on scrutinizing the videos, it was highly unusual for the dog to leave and re-enter even in a six, nine or twelve second activation). Given the large number of activations, and the potential for significant error in estimating the exact moment a dog focused on, and focused away from, a screen, instances of looking at the screen were counted, as opposed to counts being made of the time spent looking at the screen. This method, whilst losing some data fidelity, allowed us to assess the feasibility of the DoggyVision system and to make robust comparisons across dogs and conditions (week 1 vs. week 2).

As the data is not independent, and is pilot data with just two subjects $(N=2)$, only descriptive statistics (counts, frequencies, standard deviations (SD) and means (M)) are used to show the main results from the field study. All analyses were performed in SPSS version 25.

\section{Results}

The results below are presented according to our two aims; the first of evaluating the DoggyVision system in regard to the amount of attention the dogs paid to it, the workability, usefulness and suitability of the system; and the second which was to discover whether learning took place, focusing on any changes between the weeks in the dogs' looking and non-looking behavior.

As the system was in place for 14 days, it was possible to track the dogs' activations over time (Figure 4). The tracking was expected to expose two key findings; firstly - whether the dog was looking at the media when it was activated (which gives a sense of the usability of the DoggyVision system) and secondly, whether there was any evidence that the dog learned to use the system.

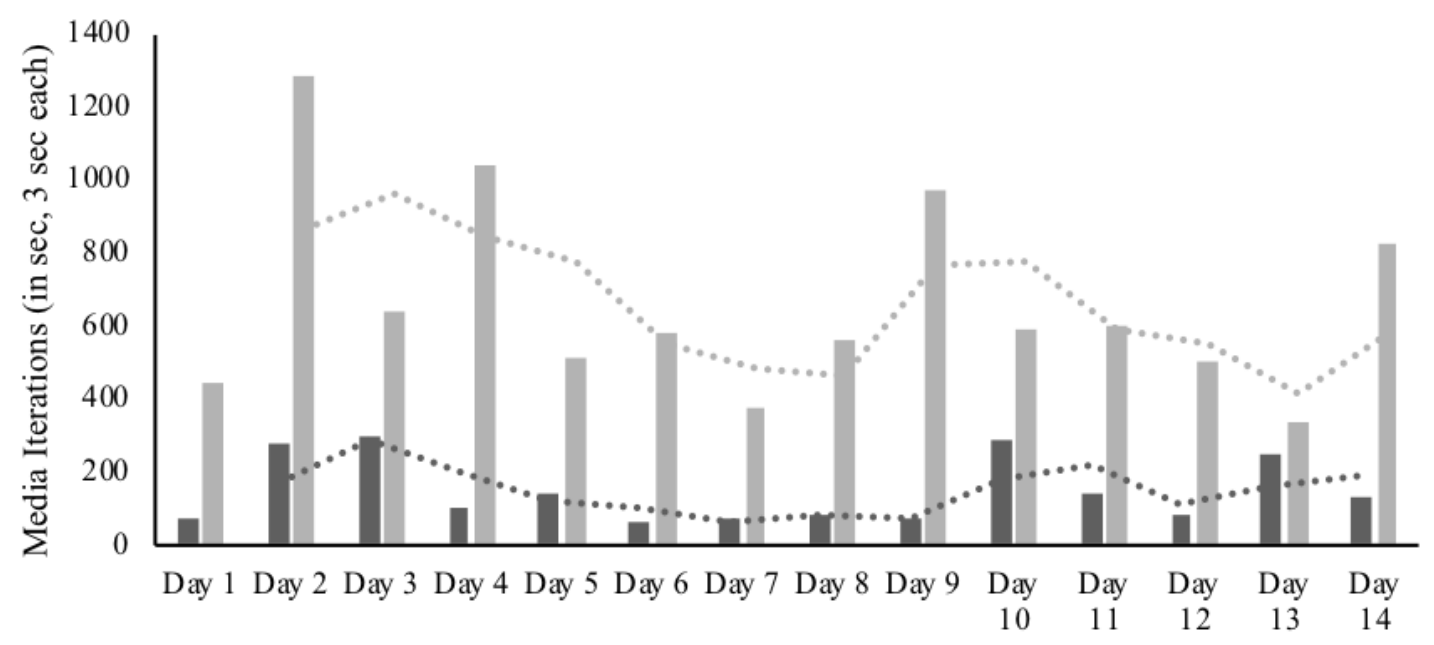

Day (1-7 Baseline [Mode 1]; 8-14 Device with Screen [Mode 2])

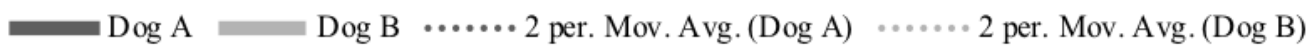

Figure 4. Dog A and Dog B media iterations over the 14-day study period (1-7 days without the screen turned on and 8-14 with the screen turned on) with a 2 point moving average trend line. 


\section{Evaluation of the System}

With regard to the amount of attention the dogs paid to the device, from the Tables 1 - 3, we can determine that the dogs looked towards the dog screen device when placed within their home if it was playing media. However, the results also indicate that, for much of the time, the dogs did not attend to the screen even when it was on (they attended as little as $21 \%$ for Dog B and 23\% for Dog A; Table 3).

Table 1

Summary of DoggyVision Data with Dog A.

\begin{tabular}{lccc}
\hline & Week 1 & Week 2 & Difference \\
\hline Dog-Activations & 490 & 497 & +7 \\
Average number of dog-activations Per Day & 70 & 71 & +1 \\
Not Looking & 489 & 382 & -107 \\
Looking Activations & 1 & 115 & +114 \\
Longest Activation (media iterations of 3 seconds) & 18 & 18 & 0 \\
Shortest Activation (media iterations of 3 seconds) & 1 & 1 & 0 \\
Mean (media iteration of 3 seconds) & 1 & 1 & 0 \\
\hline
\end{tabular}

Note. A media activation is the total activation containing cycles of media iteration (3 seconds each)

Table 2

Summary of DoggyVision Data with Dog B.

\begin{tabular}{lccc}
\hline & Week 1 & Week 2 & Difference \\
\hline Dog-Activations & 2079 & 1841 & -238 \\
Average number of dog-activations Per Day & 297 & 263 & -34 \\
Not Looking & 2076 & 1459 & -617 \\
Looking Activations & 3 & 382 & +379 \\
Longest Activation (media iterations of 3 seconds) & 17 & 12 & -5 \\
Shortest Activation (media iterations of 3 seconds) & 1 & 1 & 0 \\
Mean (media iteration of 3 seconds) & 1 & 1 & 0 \\
\hline
\end{tabular}

Mean (media iteration of 3 seconds)

Note. A media activation is the total activation containing cycles of media iteration (3 seconds each)

Table 3

Percentage of Looking and Not Looking Activations.

\begin{tabular}{clcc}
\hline \multicolumn{2}{c}{} & & Week 1 \\
\hline \multirow{2}{*}{ Dog A } & Looking & $0.20 \%$ & $23.14 \%$ \\
& Not Looking & $99.80 \%$ & $76.86 \%$ \\
\hline \multirow{2}{*}{ Dog B } & Looking & $0.14 \%$ & $20.75 \%$ \\
& Not Looking & $99.86 \%$ & $79.25 \%$ \\
\hline
\end{tabular}

As seen in Figure 4, Dog A and Dog B's activation times varied daily most probably as a consequence of the rhythms of the home. Over the two weeks, Dog A went into the activation space in a similar pattern from week one to week two, whereas Dog B's time in front of the device decreased from week one to week two (Figure 4), and overall showed occasional daily changes in activations, which varied with no set pattern.

Over the two weeks, Dog A had on average of 71 activations per day within the device space, Dog $\mathrm{B}$ had a higher average number of 297 (the total number of activations for the two weeks were Dog $\mathrm{A}=$ 987, and Dog B = 3920; Tables 1 \& 2). We hypothesize that the difference between the two dogs' activation frequencies may be due to age differences and the activity levels of the dogs; Dog A was noted as calmer (and was older) and Dog B was younger and regarded as very active by the owner. However, testing with other dogs of various ages would be necessary to investigate associations with age and personality further. One other factor that could influence the number of dog activations was the layout of the home; it could be that Dog A was generally less inclined to go into the room with the DoggyVision device than Dog B was. What this data seems to suggest is that the deployed system did not present an 'attraction' to the dog in so far as drawing it into the activation space when the video played. 
Methodologically, the data shows the need for baseline data in order to understand how the rhythm of daily life in a house might affect such a research study.

When we consider only the activations when the media was playing, the results also show, similarly to those of Hirskyj-Douglas et al. (2017), that overall, the dogs preferred to not watch the screen. When coding the videos captured by the DoggyVision camera, that non-attendance to a played screen was often seen when the dog was already pre-occupied, such as running around with a toy, preparing for a walk, or chewing things. These behaviors, although not studied here, may be worth further study as they would add to the understanding of dogs interacting with at home screen media.

During coding, and also as reported by the owners, there were no negative behavior events identified towards the device. Enabling a choice for the dogs to ignore the device probably helped in this regard as this approach enabled the dog to engage in other behaviors, without penalty, even when the media clips were playing. Dogs have been shown in our previous studies (Hirskyj-Douglas et al., 2017) to spend the majority of their time ignoring media being played; with the dog-driven interface we had expected this to not be the case (as mentioned in our predicted results).

Examining the videos, it was noted that both dogs would often go through periods of 'looking' at the screen before going back to 'not looking' for a certain period; these patterns appeared 'intentional' rather than random but due to the fact that there were only two subjects, and due to the relatively short amount of time that the dogs did 'look at' the media, this suggestion would need further study. Interestingly, the longest activations the dogs had with DoggyVision often seemed to be where the owners were present ${ }^{2}$. This gives further research directions for ACI to explore how the owners' use of a system changes their dog's use. A further dimensionality of this data would be to encode the activations when the owner was present and absent and include the proportion where the dog was looking and not looking at the device in these two cases.

\section{Increase in Activations and/or Looking Behavior over Time}

The results displayed in Table 1 indicate that, over the two weeks of the study, Dog A behaved quite similarly each day with little change in the average number of daily activations between week 1 and week 2 (7 more activations; Table 1), thereby giving no real indication that the dog learned to activate the DoggyVision system. Unsurprisingly, given that dogs are known to attend to media, Dog A looked at the screen much more when the screen was displaying media (week 2), than when no media was played (Table 3). Investigating further into Dog A's looking behavior, Dog A significantly increased his looking ratio per activation from week $1(M=0.001, S D=0.003)$ to week $2(M=0.234, S D=0.127)($ Table 4$)$ giving further evidence that dogs will attend towards media when it is being shown.

Dog B had a similar percentage of screen watching (looking media iterations) to Dog A (Table 3; Week 2, Dog A 23\% Dog B 21\%). The number of dog-activations went down slightly but not significantly for Dog B from week one $(M=303.57, S D=69.20)$ to week two $(M=257.29, S D=130.63)$ (238 less activations; Table 2), which may suggest that the dog chose to avoid the area so as to not activate the system and could therefore suggest some evidence of learning behaviors. As with Dog A, Dog B looked at the screen more when the screen was playing media, in comparison to the baseline (when no media was being played [week 1]) and, in fact, both dogs presented more 'looking' behavior during week 2 than in week 1. Dog A had a 22.94\% increase in looking behaviors and Dog B a 20.61\% increase (Table 3). Similarly to Dog A, Dog B significantly increased his looking behavior ratio per activation between week $1(M=0.001, S D=0.003)$ and week $2(M=0.24, S D=0.13$, Table 4$)$.

\footnotetext{
${ }^{2}$ The dog owners did not report a notable change in their dogs' behavior when the DoggyVision system was activated. Although they commented on how they noticed their dog watching the DoggyVision screen, in their opinion, they did not think that their dogs consciously tried to activate the DoggyVision tracker.
} 
Table 4

Ratio of Looking Behavior per Activation Between Week 1 and Week 2 with DoggyVision for Dog A and Dog B.

\begin{tabular}{lccc}
\hline & & Dog A & Dog B \\
\hline Week 1 & Day 1 & 0 & 0.004 \\
& Day 2 & 0 & 0.008 \\
Day 3 & 0.009 & 0 \\
Day 4 & 0 & 0 \\
Day 5 & 0 & 0 \\
Day 6 & 0 & 0 \\
Week 2 & Day 7 & 0 & 0.1 \\
& Day 8 & 0.296 & 0.211 \\
& Day 9 & 0.358 & 0.109 \\
& Day 10 & 0.301 & 0.066 \\
Day 11 & 0.126 & 0.189 \\
Day 12 & 0.389 & 0.235 \\
Day 13 & 0.15 & 0.542 \\
Day 14 & 0.059 & 0.315 \\
\hline
\end{tabular}

To explore our second aim of examining whether learning took place, the percentage of 'Looking' behaviors were examined over the second week on a day-to-day basis (Figure 5). As the results show, there was no trend towards an overall increase in the dogs' looking behaviors; however, both dogs did increase and decrease their looking behaviors throughout the second week when the screen was activated, and both had a peak of looking behavior on Day 6. It is not clear why there is this peak; both dogs started the study on different days, so it was not a weekend or anything similar - and there was nothing special about the owners happening on that day, so it could just be coincidence. The only way to examine trends further would be to leave the DoggyVision system in the house for longer.

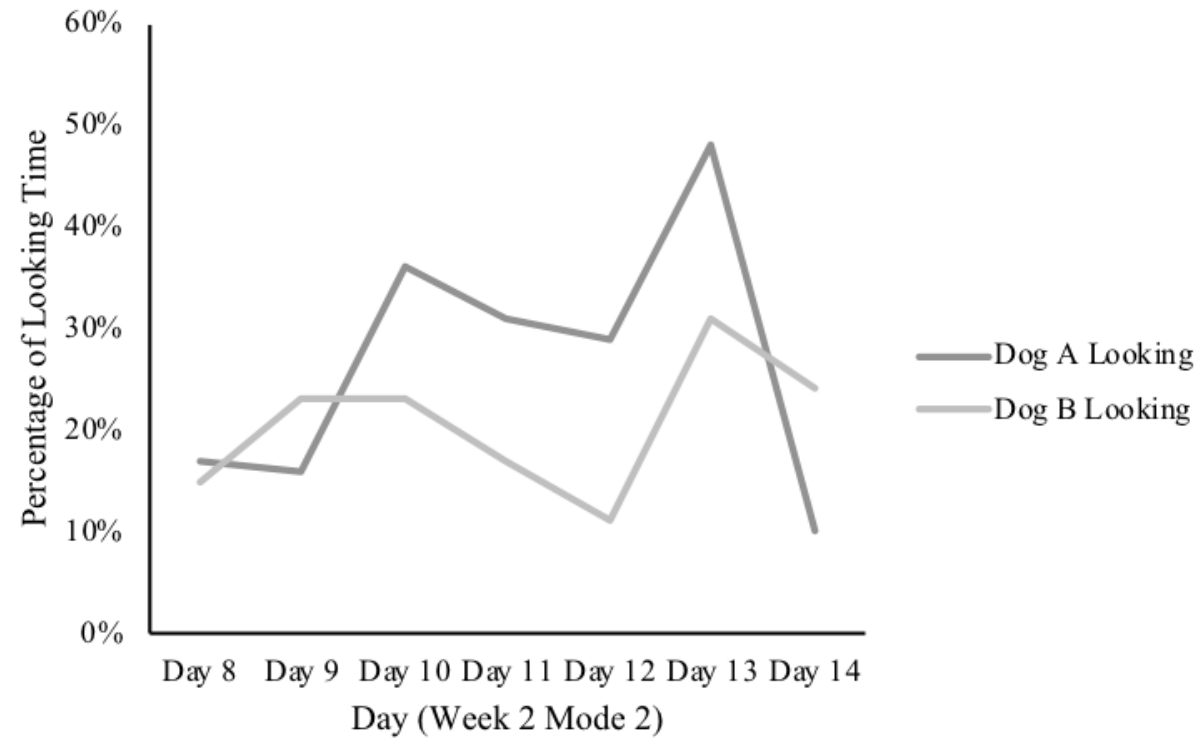

Figure 5. Percentage of looking activations split through the 7-day period when DoggyVision was on mode 2 (screen active) for Dog A and $\operatorname{dog}$ B. Percentages were rounded to the nearest whole number. 


\section{Discussion}

The findings from this study contribute to knowledge in terms of methods to study dogs' interactions with screen media and knowledge about how dogs attend to screen media in their natural environments - the home. The following discussion will explore these two themes and will present limitations, and suggestions for future work while situating the results found here with others in animal computing.

\section{What was Learned about the Method}

The method used to record dogs' activations with the screen device was effective at gathering useful results in a user centered manner. We did not find any negative behaviors directed towards the DoggyVision device, indicating that the dogs were comfortable with the device.

Both dogs showed similar behaviors when interacting with the device; notably, they would often move around, and not stand still. In addition, they would often wag their tails, run in and out of the activation space or have long periods within the space (sleeping) and generally behaving normally. Positioning the DoggyVision tracker at the dogs' body level to allow sleeping (laying down) in the activation area, and to permit the fast wagging of a dog's tail made for a user centered system. In this manner, the device functionalities (as mentioned within the design and ethical decision section above) were designed to fit the dogs' normal behavior. This design takes a step forward for animal computing, that HCI has already taken, of optimizing products around the user rather than requiring the user to change their behavior (noted in training scenarios as used often in animal science). The method of using proximity measurements as an input space gave the dogs a natural method of activation that adhered to the principles outlined by Mancini (2013) and Hirskyj-Douglas and Read (2016b), to bring a dog friendly method to the ACI community. The stance of designing for the dogs' interaction style by allowing exploration of a device from a dog's perspective, and then using the results given by the dog to further tune the device, is what forms the basis of dog-friendly research.

Manually checking each video to code whether the dog was looking towards the screen did take time. It would be good to be able to automate this process in a future version of the software and the system. Additionally, the manual checking was used to eliminate human activations from the video and system data. Despite the extra time needed to process the data, the automated recording did allow the system to run in a natural setting (within the home) over a considerable length of time, which would not have been possible in a laboratory environment.

The baseline recording in week one was shown to be very important as evidenced by the different activity levels of the two dogs. Without this data, misinterpretations of the dogs' activations and looking behaviors in week two could have occurred. Similarly, the choice to log each day over a week was crucial as the dogs' daily patterns changed considerably according to their routines. By additionally videoing the dogs during the activations, we gained useful information that enabled us to understand what an interaction was made up of, we could screen out human activations and this also allowed the collection of rich data in the home showing how dogs entered and left the tracked area.

\section{What was Learned about Dogs and Screens}

One of the key contributions to come from this study was the development of the DoggyVision system that theoretically could allow a dog to control their activation of a device. The DoggyVision system was shown to work with different dogs over a longitudinal period without human intervention.

The activations caused by the dogs changed daily in dissimilar patterns for unknown reasons with the dogs appearing to go through high and low periods of interactivity, sometimes setting off the device intermittently and sometimes continually. This could be for many reasons including, that the dogs were 
continually within the activation space, the dogs remaining to watch the changing media clips, or exploring the device in general. Even the weather outside could have affected the in-home behavior.

When analyzing the longest activations that the dogs had with DoggyVision when it was playing media clips, the two dogs behaved differently but shared similar behaviors of ear pricking and head movements. This begins to bring researchers insight into how dogs use devices and shows that, although the dogs were not always watching the system as it played media clips, their attention did flicker between the environment, other modalities and the screen. Whereas dog media devices like these have been suggested for use when a dog is home alone, it is worth mentioning that the dogs' longest activations were each when the dog was with the owner. Whether the owner was present during the DoggyVision activations was not coded within the schema used within this analysis; however, the video allowed us to note if the owner was present. There is scope within this method to factor in the owners' possible influence on the dog's usage of the devise, by coding owner presence and absence and examining whether the frequency of activations and looking times varied. The current study highlights the need to investigate further the human's role, and influence, in animal robotic systems.

Overall, the dogs preferred not to watch the media, looking elsewhere, as the DoggyVision device was delivering media content. As discussed within ACI, this is not the first instance of ACI technology being ignored by animals (Hirskyj-Douglas et al., 2017; Ritvo \& Allison, 2014). This does bring to question the definition of 'interactivity' for dogs, that in terms of whether interactive screen devices need to be watched to be classed as interactive (as shown here through 'looking' codes). As an example, many owners will often leave the radio on for dogs, when leaving them at home alone (Takeuchi, Houpt, \& Scarlett, 2000); therefore, we might consider that, in the current study, the dogs, when facing away from the media, might have been attending to the device, albeit not 'looking'. In addition, the attention of the dog to the device could have been a natural reflex reaction to the sound and visual stimulus, as shown by ear pricking, or could have been the dog's self-initiated behavior; the latter could have been considered intentional. We aimed to minimize reflex responses through lowering the system's sound volume, but its impact is unknown. This behavioral difference could be further considered by comparing the standard behavior of the dog when watching TV to the DoggyVision device.

\section{Limitations}

A major limitation of this work is the small sample size; however, our study provides a crucial step towards producing working dog-driven screen devices. As only two dogs were tested, further investigations to validate this system on various dogs, homes, and owners would be necessary. The limitations of the method were mainly around the tracking of the dogs' entry into and exit from the activation space, which had to be optimized to fit with the dog's ordinary movements. Thus, the three second counter was used and therefore any rapid movements in and out of the activation area were discounted during this time. A more sophisticated detection system, such as face and/or body recognition as used in HCI, could be designed to allow more granularity of tracking.

\section{Future Directions}

As the hardware and software of the system worked well, there is potential for this prototype to be further developed. An interesting divergence would be to test DoggyVision with a different activation method other than proximity, such as using tactile devices or changing the screen to an 'on the floor' projected interactive system. As screen devices evolve, with virtual reality becoming a possibility for dogs through visual interface screens (Ohta, 2016) and TV viewing changing over the years (Wheatley, 2014), this method could be used with different screens or devices. In addition, more work would need to be carried out to explore the dogs' variation in use and to ascertain if, given enough time, the dogs could properly learn to activate the system opening up the space into new interaction methods.

The varying frequency of interactions could be due to the dogs' age as several studies have reported decreases in activity levels with age in dogs within the home environment. This decrease is 
sometimes associated with cognitive function (Landsberg, Nichol, \& Araujo, 2012) and locomotor activities (Siwak, Murphey, Muggenburg, \& Milgram, 2002). Further work could consider age but also the personality of the dog and other attributes that might affect the dog's interaction frequencies. Frameworks have been suggested to measure a dog's personality using owner questionnaires (Gosling, Kwan, \& John, 2003), and distinct personality types might result in higher interaction rates; for example, dogs scoring highly in activity and excitability or trainability might have higher interaction rates. It would also be interesting to study further which media clips dogs find interesting and rewarding, extending work by Williams et al. (2011), Törnqvist et al. (2015), and Somppi et al. (2012). The DoggyVision system, with its non-training stance could be especially insightful over a longitudinal study. Because there were only two dogs tested here, it could be that these happened to be dogs that were not motivated to look at screens and thus, this phenomenon is open for further investigation. Additionally, as was investigated here, the dogs' activity could be further explored using supplementary devices, such as activity monitors, as a further control for dogs' levels of daily activity and response. The benefits of such technology could also potentially be measured through hormone measurements such as cortisol levels (Geurtsen et al., 2015) and behaviors such as decreased stress behaviors and increased rest behaviors. Finally, through further iterations of this study, a corpus of dog movements with an interactive screen device could be built up; this would be ideal to train devices to detect natural behaviors thus reducing the training needed for dogs to use computer systems and better allowing systems to be shaped around the dog's affordances and requirements. This device could also potentially be adjusted for each dog creating personalized systems as suggests by employing a standard tracking device to learn and then be individualized to adjust for each dog, and thus aim towards a user centered design.

\section{Conclusions}

We aimed to determine if we could design a system that would allow dogs to use an interactive media device within their own home (aim 1) and to explore if, with such a device they would learn to activate it themselves (aim 2). A system was designed, DoggyVision, and put into dogs' homes allowing dogs to initiate their own media activations, recording viewing habits between an on and off state independent of the human researcher. The implemented system includes: (1) tracking: a way of tracking dogs to enable a device to turn on and off, (2) media delivery: when a dog is detected, a media clip is randomly selected and then played automatically, (3) recording: once activated the system begins video recording the subject, and lastly (4) baseline and active: the system monitors activations firstly by creating a baseline when no media is presented, and then in a second mode, when media is presented. In the case of the first aim, our results show that the dogs attended to, and were able to use, the system with both dogs noticing, activating and looking at the device. Towards the second aim, although there were minor changes in the number of times the dogs activated the device there were increases in looking times in week 2 suggesting that the dogs were interested in the media, but did not immediately habituate to it, and therefore, it is unclear if learning had taken place. Overall, even though the device was designed specifically for dogs, the dogs, when confronted with the device for a long period, either behaved similarly to the baseline or avoided the device preferring not to watch the media presentations.

The insights brought by this study would benefit those seeking to develop dog-interactive media products and those working in ACI with screen devices. The work here builds upon previous methods helping to construct a set of dog-centric interaction methods in ACI by bringing a way of investigating animal-screen activation. Importantly, the method of a baseline data is introduced and implemented to provide a way of assessing variables in a field study space where animals currently reside. Our methodology is the first system, that the authors know of, that potentially allows a dog to initiate its own media viewing without being trained to do so. Overall, this study highlights the importance of exploring dogs' use of devices by using an ethogram of behavior through allowing the animal itself to explore technology. These contributions whilst pertinent to the design of interactive technology for dogs, also present guidelines for researchers working with dogs, highlighting the importance of ethics when utilizing screen technologies in the home. 


\section{Acknowledgements}

We are grateful to Dr Brendan Cassidy for his assistance in the construction of the program along with Dr. Daniel Fitton who also aided in this aspect. We would like to thank the three anonymous reviewers, and Dr. Judit Abdai whose comments improved many parts of the paper.

\section{References}

Abreu, J., Almeida, P., \& Teles, B. (2014). TV discovery \& enjoy: A new approach to help users finding the right TV program to watch. In Proceedings of the ACM International Conference on Interactive Experiences for TV and Online Video (pp. 63-70). ACM.

Britt, W., Miller, J., Waggoner, P., Bevly, D., \& Hamilton, J. (2011). An embedded system for real-time navigation and remote command of a trained canine. Personal and Ubiquitous Computing, 15, 61-74.

Buchanan, R. (2001). Design research and the new learning. Design Issue, 17, 3-23.

Carter, M., Webber, S., \& Sherwen, S. (2015). Naturalism and ACI: Augmenting zoo enclosures with digital technology. Paper presented at 12th International Conference on Advances in Computer Entertainment Technology (p. 61). Malaysia. doi: 10.1145/2832932.2837011

Doggen. J. (2014). Sharp IR sensors library for arduino. Arduino playground. Arduino Playground. Retrieved from http://playground.arduino.cc/Main/SharpIR

DogTV (2016). DogTV. DogTV. Retrieved from http://dogtv.com

Freil, L., Byrne, C., Valentin, G., Zeagler, C., Roberts, D., Starner, T., \& Jackson, M. (2017). Canine-centered computing. Foundations and Trends in Human-Computer Interaction, 10, 87-164.

French, F., Mancini, C., \& Sharp, H. (2015). Designing interactive toys for elephants. In CHI PLAY '15: Proceedings of the 2015 Annual Symposium on Computer-Human Interaction in Play, ACM (pp. 523-528)

Gosling, S. D., Kwan, V. S., \& John, O. P. (2003). A dog's got personality: A cross-species comparative approach to personality judgments in dogs and humans. Journal of Personality and Social Psychology, 85, 1161-1169.

Geurtsen, A., Lamers, M. H., \& Schaaf, M. J. (2015). Interactive digital gameplay can lower stress hormone levels in home alone dogs - A case for animal welfare informatics. In K. Chorianopoulos et al. (Eds.), Entertainment Computing-ICEC. (pp. 238-251). Springer.

Haahr, M. (2016). Random number generator. Retrieved from https://www.random.org/.

Hirskyj-Douglas, I., Huailan L., \& Read, J. C. (2014, October). Is my dog watching TV? Paper presented at NordiCHI'14 Workshop on Animal Computer Interaction. Helsinki, Finland.

Hirskyj-Douglas, I., \& Read, J. C. (2014). Who is really in the center of dog computer interaction? Paper presented at Advance Computer Entertainment (ACE)'14. Madeira: ACM.

Hirskyj-Douglas, I. \& Read, J. C. (2016a). Using behavioral information to help owners gather requirements from their dogs' responses to media technology. In The 30th International BCS Human Computer Interaction Conference: Fusion! (pp. 42-55). BCS Learning \& Development Ltd.

Hirskyj-Douglas, I., \& Read, J. C. (2016b, May 26th). Ethics of how to work with dogs in animal computer interaction. Paper presented at Measuring Behavior Symposium on Animal Computer Interaction, Dublin, Ireland.

Hirskyj-Douglas, I., Read, J. C., \& Cassidy, B. (2017). A dog centred approach to the analysis of dogs' interactions with media on TV screens. International Journal of Human-Computer Studies, 98, 208-220.

Issa, T., \& Isaias, P. (2015). Usability and human computer interaction (HCI). In T. Issa \& P. Isaias (Eds.), Sustainable design (pp. 19-36). London: Springer.

Lakatos, G., Gácsi, M., Topál, J., \& Miklósi, Á. (2012). Comprehension and utilisation of pointing gestures and gazing in dog-human communication in relatively complex situations. Animal Cognition, 15, 201-213.

Landsberg, G. M., Nichol, J., \& Araujo, J. A. (2012). Cognitive dysfunction syndrome: A disease of canine and feline brain aging. Veterinary Clinics: Small Animal Practice, 42, 749-768.

Lee, P., Cheok, D., James, S., Debra, L., Jie, W., Chuang, W. \& Farbiz, F. (2006). A mobile pet wearable computer and mixed reality system for human-poultry interaction through the internet. Personal and Ubiquitous Computing, 10, 301-317.

Lemasson, G., Duhaut, D., \& Pesty, S. (2015, July). Dog: Can you feel it? Paper presented at Animal Computer Interaction@ British Human Computer Interaction (BHCI), Lincoln, England. 
Lim, Y. K., Donaldson, J., Jung, H., Kunz, B., Royer, D., Ramalingam, S., \& Stolterman, E. (2008). Emotional experience and interaction design. In C. Peter \& R. Beale (Eds), Affect and emotion in human-computer interaction (pp. 116-129). Berlin, Heidelberg: Springer.

Majikes, J., Brugarolas, R., Winters, M., Yuschak, S., Mealin, S., Walker, K., \& Roberts, D. L. (2017). Balancing noise sensitivity, response latency, and posture accuracy for a computer-assisted canine posture training system. International Journal of Human-Computer Studies, 98, 179-195.

Mancini, C. (2011). Animal-computer interaction: A manifesto. Interactions, 18, 69-73.

Mancini, C. (2013). Animal-computer interaction (ACI): Changing perspective on HCI, participation and sustainability. In CHI'13 Extended Abstracts on Human Factors in Computing Systems (pp. 2227-2236). Paris, France.

Mancini, C. (2016). Towards an animal-centred ethics for animal-computer interaction. International Journal of Human-Computer Studies, 98, 221-233.

Mancini, C., Harris, R., Aengenheister, B., \& Guest, C. (2015). Re-centering multispecies practices: A canine interface for cancer detection dogs. The 33rd Annual ACM Conference on Human Factors in Computing Systems (pp. 2673-2682). ACM.

Mancini, C., Lawson, S., \& Juhlin, O. (2016). Animal-computer interaction: The emergence of a discipline. International Journal of Human-Computer Studies, 98, 129 - 134.

Mealin, S., Domínguez, I. X., \& Roberts, D. L. (2016). Semi-supervised classification of static canine postures using the Microsoft Kinect. In Proceedings of the Third International Conference on Animal-Computer Interaction (p. 16).

Mealin, S., Winters, M., Domınguez, I. X., Marrero-Garcıa, M., Bozkurt, A., Sherman, B. L., \& Roberts, D. L. (2015). Towards the non-visual monitoring of canine physiology in real-time by blind handlers. In Proceedings of the 12th International Conference on Advances in Computer Entertainment Technology (p. 66).

Morrison, A., Møller, R.H., Manresa-Yee, C., \& Eshraghi, N. (2016). The impact of training approaches on experimental setup and design of wearable vibrotactiles for hunting dogs. In Proceedings of the Third International Conference on Animal-Computer Interaction (p. 4).

Norman, D. (1988). The design of everyday things. Doubleday, New York.

Ohta, N. (2016, May). Animal-human digital interface: Can animals collaborate with artificial presences? Presented at Measuring Behavior Symposium on Animal Computer Interaction: Dublin, Ireland.

Olsen, D., Sellers, B., \& Boulter, T. (2014, June). Enhancing interactive television news. In Proceedings of the 2014 ACM International Conference on Interactive Experiences for TV and Online Video (pp. 11-18).

PDSA PAW Report. (2015). PDSA Animal Wellbeing (PAW) Report. Retrieved from https://www.pdsa.org.uk/ /media/pdsa/files/pdfs/veterinary/paw-reports/pdsa-paw-report-2015.ashx?la=en

Perdue, B. M., Clay, A. W., Gaalema, D. E., Maple, T. L., \& Stoinski, T. S. (2012). Technology at the zoo: The influence of a touchscreen computer on orangutans and zoo visitors. Zoo Biology, 31, 27-39.

Piotti, P., Spooner, R. M., Jim, H. L., \& Kaminski, J. (2017). Who to ask for help? Do dogs form an opinion on humans based on skilfulness? Applied Animal Behavior Science, 195, 93-102.

Pongrácz, P., Miklósi, A., Kubinyi, E., Gurobi, K., Topál, J., \& Csányi, V. (2001). Social learning in dogs: The effect of a human demonstrator on the performance of dogs in a detour task. Animal Behavior, 62, 11091117.

Raynor, H. A., Steeves, E. A., Bassett, D. R., Thompson, D. L., Gorin, A. A., \& Bond, D. S. (2013). Reducing TV watching during adult obesity treatment: Two pilot randomized controlled trials. Behavior Therapy, 44, 674-685.

Resner, B. I. (2001). Rover@ Home: Computer mediated remote interaction between humans and dogs. (Unpublished doctoral dissertation). Massachusetts Institute of Technology, Cambridge, MA, USA.

Ritvo, S., \& Allison, R. (2014). Challenges related to nonhuman animal-computer interaction: Usability and 'liking'. In Proceedings of the 2014 Workshop on Advances in Computer Entertainment Conference, 11-14. ACM.

Robinson, C. L., Mancini, C., Van Der Linden, J., Guest, C., \& Harris, R. (2014). Canine-centerd interface design: Supporting the work of diabetes alert dogs. The 32nd annual ACM Conference on Human Factors in Computing Systems. (pp. 3757-3766).

Siwak, C. T., Murphey, H. L., Muggenburg, B. A., \& Milgram, N. W. (2002). Age-dependent decline in locomotor activity in dogs is environment specific. Physiology \& Behavior, 75, 65-70.

Smith, J. (2011). Apps for apes: Orang-utans want iPads for Christmas. New Scientist, 212, 69-71.

Somppi, S., Törnqvist, H., Hänninen, L., Krause, C., \& Vainio, O. (2012). Dogs do look at images: Eye tracking in canine cognition research. Animal Cognition, 15, 163-174. 
Soproni, K., Miklósi, Á., Topál, J., \& Csányi, V. (2001). Comprehension of human communicative signs in pet dogs (Canis familiaris). Journal of Comparative Psychology, 115, 122-126.

Takeuchi, Y., Houpt, K. A., \& Scarlett, J. M. (2000). Evaluation of treatments for separation anxiety in dogs. Journal of the American Veterinary Medical Association, 217, 342-345.

Topál, J., Byrne, R. W., Miklósi, A., \& Csányi, V. (2006). Reproducing human actions and action sequences: "Do as I Do!" in a dog. Animal Cognition, 9, 355-367.

Törnqvist, H., Somppi, S., Koskela, A., Krause, C. M., Vainio, O., \& Kujala, M.V. (2015). Comparison of dogs and humans in visual scanning of social interaction. Royal Society Open Science, 2, 150341.

Trindade, R., Sousa, M., Hart, C., Vieira, N., Rodrigues, R., \& França, J. (2015). Purrfect crime: Exploring animal computer interaction through a digital game for humans and cats. In Proceeding of the 33rd Annual ACM Conference Extended Abstracts on Human Factors in Computing Systems (pp. 93-96).

Valentin, G., Alcaidinho, J., Howard, A., Jackson, M. M., \& Starner, T. (2015). Towards a canine-human communication system based on head gestures. The 12th International Conference on Advances in Computer Entertainment Technology. ACM.

Westerlaken, M., \& Gualeni, S. (2014). Felino: The philosophical practice of making an interspecies videogame. In Proceedings of the Philosophy of Computer Games Conference, Istanbul, Turkey.

Wheatley, D. J. (2014). Design and evaluation of a children's tablet video application. The 2014 ACM International Conference on Interactive Experiences for TV and Online Video, 79-86.

Williams, F. J., Mills, D. S., \& Guo, K. (2011). Development of a head mounted, eye-tracking system for dogs. Journal of Neuroscience Methods. 194, 259-265.

Zeagler, C., Zuerndorfer, J., Lau, A., Freil, L., Gilliland, S., Starner, T., \& Jackson, M. M. (2016). Canine computer interaction: Towards designing a touchscreen interface for working dogs. The Third International Conference on Animal-Computer Interaction. 